\title{
Corela
}

Cognition, représentation, langage

HS-4 | 2006

Le parcours

\section{Comment référer à une classe ? Variations autour du concept de PARCOURS}

\section{Lucie Gournay}

\section{OpenEdition}

\section{Journals}

Édition électronique

URL : http://journals.openedition.org/corela/1336

DOI : 10.4000/corela.1336

ISSN : $1638-573 \mathrm{X}$

\section{Éditeur}

Cercle linguistique du Centre et de I'Ouest - CerLICO

Référence électronique

Lucie Gournay, "Comment référer à une classe ? Variations autour du concept de PARCOURS », Corela [En ligne], HS-4 | 2006, mis en ligne le 08 juin 2006, consulté le 01 mai 2019. URL : http:// journals.openedition.org/corela/1336; DOI : 10.4000/corela.1336

Ce document a été généré automatiquement le 1 mai 2019.

\section{(c) (i) (2)(2)}

Corela - cognition, représentation, langage est mis à disposition selon les termes de la licence Creative Commons Attribution - Pas d'Utilisation Commerciale - Partage dans les Mêmes Conditions 4.0 International. 


\title{
Comment référer à une classe? Variations autour du concept de PARCOURS
}

\author{
Lucie Gournay
}

\section{Le problème avec le parcours}

1 Dès qu'on essaye d'étudier de près la question du parcours, on s'aperçoit qu'il y a plusieurs définitions qui circulent plus ou moins implicitement dans les articles de Culioli $(1990,1999 a, 1999 b)^{1}$, des définitions qui ne s'opposent jamais franchement, mais qui ont des aspects contradictoire ce qui rend difficile l'identification des marqueurs de parcours.

A titre d'exemple, les deux citations suivantes:

«Le parcours à la différence de l'extraction et du fléchage, fait que l'on ne peut (ou veut) distinguer aucune occurrence dans le domaine » (Culioli 1990 : 121)

« as for Scanning, it is in a class of its own, as far as individuating operations are concerned, although it includes modes of Scanning that relate to Extraction and Pinpointing. » (Culioli 1990 : 183)

2 Si je compare ces deux définitions je ne sais plus si le parcours est en opposition avec l'extraction (1ère définition) ou non (2ème définition). Par ailleurs, il existe d'autres symptômes de cette « crise » du parcours.

\section{1. mention « parcours abstrait !»}

3 Culioli (1990: 111) nous rappelle que lorsqu'il parle de parcours, il s'agit d'un parcours abstrait, ce qui montre bien qu'il est conscient des pièges de sa métalangue. Avec les termes de "parcours", «extraction", «fléchage » pour renvoyer à des opérations de déterminations, on "voit» des gestes et on en oublie fatalement la réalité formelle représentée, qui est, elle, beaucoup moins intuitive. Ce qui fait que pour certains auteurs parcours et itération sont synonymes et signifient " passer en revue un champ temporel » 
comme dans le cas des récurrences d'évènements. A quand le parcours renvoyant à une changement de localisation?

\subsection{Une multiplication de parcours}

4 Dans le cas du parcours, uniquement, on assiste à une inflation des types définis (certains étant en fait sont synonymes) A titre d'exemple, voici ce qu'on trouve dans les notes de séminaires de Culioli 1983-84 et dans Pour une linguistique de l'énonciation: parcours quantitatif, parcours qualitatif, parcours centré, parcours avec ou sans issue/sortie, parcours avec totalisation, parcours avec extraction, parcours lisse, parcours rugueux, parcours moiré, parcours avec image, parcours strict, parcours-parcours.

Et la liste s'agrandit encore si l'on prend en compte d'autres auteurs dans cette même théorie...

5 Cette multiplication m'amène à penser qu'il y a une différence entre la définition généralement donnée (Culioli 1990: 121), qui définit une opération en opposition à l'extraction et au fléchage, et le concept plus général de parcours utilisé ici et là. Celui-là serait lié à un ressenti « pluriel » ou à de l'altérité (A dog is a mammal, parcours rugueux), irait de pair avec de l'indéfini (Qui est venu?, parcours avec image), et finalement serait envisagé comme le résultat d'un ensemble d'opérations (quand Culioli dit "l'interrogation est un parcours" (Notes séminaires) il ne parle pas de forme ou de marqueur à proprement parler, mais d'une combinaisons de repérages au sein de l'interrogation).

\subsection{Un parcours paradoxal}

6 Si le parcours bloque la possibilité d'une validation d'occurrence, il semble paradoxal d'envisager que des marqueurs anaphoriques de reprise soient des marqueurs de parcours. Comment renvoyer à un élément déjà validé par le biais d'un opérateur de nonvalidation? Ce paradoxe a été aussi relevé par Le Goffic (1994 34) à propos des marqueurs en QU- :

« ...parler ici d'un antécédent, c'est méconnaître précisément le fonctionnement pronominal autonome du pronom indéfini avec sa valeur de parcours : en tant que tel un parcours ne saurait avoir d'antécédent »

7 Et pour justifier la classification des marqueurs en QU- parmi les opérateurs de parcours, il évoque un cas particulier de parcours: le parcours capté, saturé dès le départ. Il me semble que cette astuce, largement argumentée par l'auteur, n'en reste pas moins peu satisfaisante du point de vue métalinguistique.

Il y existe encore un autre paradoxe selon moi. En effet, à lire toutes ces définitions, on ne sait plus si le parcours construit l'homogénéité des valeurs d'une classe, comme ce serait le cas dans Everybody knows that, ou si le parcours construit typiquement une individuation, comme dans Anybody knows that. Si any et every sont tous les deux des marqueurs de parcours (pour l'instant je ne me prononce pas), je veux définir ce parcours en fonction de ce que ces opérateurs ont en commun, dans leur travail sur la notion. Les éventuelles valeurs secondaires (homogénéisation, individuation etc.) sont déterminées quelque part mais pas nécessairement par l'opération de parcours elle-même. Rendons à l'opération de parcours sa spécificité car à force de tout appeler "parcours » ce terme perd sa fonction métalinguistique discriminante. 


\section{Nécessité d'une définition en opposition avec l'extraction}

9 Je vais défendre l'idée qu'il faut partir d'une définition du parcours qui s'oppose à l'extraction et, pour affiner ma position, je comparerai parcours, altérité, et indéfinitude.

\subsection{Avec le parcours, pas de restriction de la classe}

Il y a trois types de travail sur un domaine notionnel: le parcours, l'extraction et le fléchage. Avec le fléchage et l'extraction, on a deux opérations distinctes de sélection ou validation d'occurrences, qui selon les cas vont de pair avec une restriction qualitative de la classe. Avec le parcours, il n'y a pas de sélection, et donc pas de restriction qualitative de la classe. Justement: un marqueur de parcours signale que la sélection d'une occurrence, à partir d'un domaine discrétisé, n'est pas construite.

Tableau comparatif des 3 opérations de détermination

\begin{tabular}{|c|c|c|}
\hline $\begin{array}{l}\text { Parcours } \\
\text { Pas d'occurrence }\end{array}$ & $\begin{array}{l}\text { Extraction } \\
\text { Occurrence } \\
\text { construite }\end{array}$ & $\begin{array}{l}\text { Fléchage } \\
\text { Occurrence qualifiée }\end{array}$ \\
\hline $\begin{array}{l}\text { Délimitation QNT } \\
{[]} \\
\text { Délimitation QLT } \\
\varnothing\end{array}$ & $\begin{array}{l}\text { Délimitation QNT [ } \\
\text { ] } \\
\text { Délimitation QLT [ } \\
\text { ] }\end{array}$ & $\begin{array}{l}\text { Qualification supplémentaire QLT d'une occurrence } \\
\text { QNT/QLT déjà construite }\end{array}$ \\
\hline
\end{tabular}

11 En d'autres termes, l'extraction d'occurrence consiste à associer à une extension quantitative un ensemble de propriétés qualitatives discriminantes. Quand les propriétés qualitatives ne sont pas jugées assez discriminantes au niveau de la relation prédicative, l'extension quantitative sans délimitation qualitative associée ne permet pas la construction d'occurrence.

Regardons les exemples (1) et (2) :

(1) Any linguist would agree

(2) A linguist would agree

12 En (1), avec ANY, l'énonciateur parcourt le domaine discrétisé de LINGUIST puisqu'il signale qu'il n'y a pas de validation d'une valeur spécifique de la classe. On peut dire qu'avec ANY, on pose les propriétés de l'extension quantitative d'une seule occurrence ${ }^{2}$, et celle-ci demeure non validée car pour l'énonciateur chaque valeur de la classe est susceptible d'y apparaître. Aucune n'est distinguée des autres.

13 En (2), avec A, l'énonciateur extrait une occurrence de la classe de LINGUIST en ce sens qu'il valide un élément de la classe pour la relation prédicative donnée. Les autres valeurs de la classe ne sont pas prises en compte. On ne réfère pas à la classe. C'est le contexte prédicatif avec ses déterminations énonciatives qui construit la valeur générique 'take a linguist, he or she would agree'. 
Evidemment, avec les exemples (1) et (2), je ne cherche pas la difficulté, ayant choisi, pour illustrer ces opérations, deux marqueurs "prototypiques » de parcours et d'extraction, pour le lecteur culiolien. Je vais donc reprendre ma définition du parcours pour la confronter à des marqueurs moins consensuels.

\subsection{Le parcours et la notion d'altérité : le cas de OR}

J'ai dit plus haut que altérité et parcours allaient souvent ensemble dans la littérature TOPE. Je vais montrer que les deux termes ne sont pas synonymes.

Dans mes travaux sur la coordination, je parle de OR, opérateur de parcours, sans véritablement argumenter ce que j'avance. Aujourd'hui il me semble nécessaire de remettre cette hypothèse en question, ne serait-ce que pour distinguer entre $O R$ et EITHER...OR qui n'ont pas les mêmes distributions ${ }^{3}$.

17 Dans les deux cas, un ensemble de valeurs en opposition est exprimé, mais dans le second seulement, il y a parcours, car dans le second seulement l'énonciateur avec EITHER envisage cet ensemble de valeurs comme étant à restreindre.

18 Si on prend un énoncé comme "she is French or Belgian ", on voit bien que l'apparition de OR dans la chaîne linéaire revient à lever la validation provisoire de French, tout en introduisant une nouvelle valeur plus ou moins en opposition avec ce qui précède. OR signale que l'énonciateur met en opposition deux valeurs, mais les caractéristiques de cette opposition dépendent des propriétés des éléments opposés.

(3) I am facing the imminent destruction of all that I hold dear, or used to hold dear, anyway, and he sits there sipping his designer beer, oblivious to anything but the comfort of his surroundings and his delight in my presence. (How to be good, chapter 2)

(4) Do you have any brothers or sisters? / Is it true or false?

On le voit avec le OR à valeur corrective, comme en (3). L'énonciateur propose deux options, créant un effet d'indéfini, des deux options co-référentielles à quelque chose près. C'est notre compréhension du monde qui nous amène à interpréter cet emploi comme un OR correctif et à envisager I used to hold dear comme la bonne valeur. Mais OR n'en dit pas autant.

De même, OR, tout seul, ne dit rien sur le caractère inclusif ou exclusif de l'opposition qu'il marque. Ce sont les propriétés des arguments, comme on le voit en (4), qui donnent éventuellement une interprétation inclusive (brother/sister) ou exclusive (true/false) en fonction $\mathrm{du}$ contexte d'apparition: ailleurs, brother/sister pourraient s'opposer entièrement comme dans « Is it a sister or a brother that you have? ».

21 Si OR construit une classe de valeurs possibles, en relation d'opposition, il ne signale pas qu'à partir de cette classe, un élément et un seul serait validable dans la relation prédicative, contrairement à ANY.

22 Cependant, dans la langue, l'altérité peut être construite explicitement comme exclusive. Et là, grâce à EITHER, on obtient véritablement une opération de parcours. EITHER construit une "enveloppe vide ", c'est-à-dire une extension quantitative qui n'est pas associée à une délimitation qualitative.

(5) "You are either with us or against us" G. Bush, 6/11/ 2001

(6) I don't believe either of you.

(7) We don't enjoy seeing ourselves crudely portrayed on the movie screen either,

you know (eyeshot.net/car singer) 

occurrence est validable au niveau prédicatif. Est-ce que EITHER indépendamment de OR est un opérateur de parcours? On voit que dans ses autres emplois EITHER semble aussi fonctionner comme un opérateur de parcours : en (6) aussi, on a la délimitation d'une occurrence qui n'est pas instanciée car la distinction qualitative des valeurs auxquelles you réfère n'est pas pertinente dans la relation prédicative et que l'on a un contexte de non-validation. EITHER en fin de proposition comme en (7), liée à une relation prédicative validée à l'Extérieur (négation), qualifie cette relation en l'identifiant à une autre relation préconstruite dans la même zone extérieure.

\subsection{Parcours et « indéfinitude »: le cas de WHICH} indéfini =opérateur de parcours.

Jais prendre comme exemple WHICH qui comme les autres marqueurs simples en WH- est bien ...un marqueur d'extraction! Je le comparerai à WHICHEVER, revenant ainsi sur mes travaux récents sur $\mathrm{WH}-$, dans lesquels je montre que le classement des marqueurs en WH- comme opérateur de parcours n'est pas fondé.

En effet, l'analyse de WH- ou de QU- en terme d'image de valeurs assignables tient plus d'une perspective logique que d'un point de vue linguistique. Si l'on s'entend tous au moins pour dire qu'un parcours nécessite le renvoi à une classe de valeurs, on voit assez rapidement, que ce n'est pas le cas avec les éléments en WH- qui renvoient toujours à une occurrence qualitativement distinguée y compris dans le cadre d'une interrogation: quand je dis «WHAT?», certes mon propos est peu défini, mais j'ai quand même posé une occurrence de relation, quantitativement et qualitativement distinguée (Il y a quelque chose et quelque chose de particulier). L'assimilation $\mathrm{WH}^{-}=$parcours $^{4}$ vient $\mathrm{du}$ fait que l'on glose le sens de WH- non pas à partir de ce qui est dit mais en fonction de ce qui est susceptible d'être répondu dans le cadre d'une interrogation.

Je me suis aperçu en proposant cette analyse qu'il était plus contre intuitif de renoncer à l'analyse en parcours pour WHICH. Pourquoi?

(8) Which (of these, candidate, colour) do you prefer?

WHICH, par opposition à WHO ou WHAT, implique la reprise d'un domaine déjà construit et spécifié dans le contexte antérieur. On a donc avec le marqueur $\mathrm{WHICH}^{5}$ une indétermination de l'occurrence particularisée, alors même que l'on part d'un ensemble d'occurrences déjà définies dans le discours. C'est cette opposition entre d'une part l'indéfinitude qualitative de l'occurrence et le renvoi à un domaine spécifié (signifié par ICH) qui, peut-être, explique les hésitations avec WHICH. L'occurrence est repérée par rapport à un ensemble fini de valeurs préconstruites mais n'en demeure pas moins indéfinie.

Comme avec WHO et WHAT, WHICH construit bien une occurrence distinguée, mais qui est indéfinie dans la relation prédicative. On sait qu'il s'agit d'une occurrence particularisée, mais on ne connait pas le détail de cette particularisation. WH- signale que les propriétés qualitatives de l'occurrence sont données dans une autre lexis, soit dans le contexte antérieur (emploi pronominal) soit dans le contexte qui suit (emploi dans les questions). 
30 Avec les marqueurs en WH-, comme avec OR, il y a moyen d'introduire une opération de parcours parce qu'il y a de l'indéfini : indéfinitude sur les propriétés de l'altérité avec OR, indéfinitude avec WHICH (pour continuer avec celui-là) sur l'identification qualitative de l'occurrence dans la relation prédicative en cours.

31 Ainsi, EVER associé à WH-, construit la neutralisation de la singularité qualitative au niveau de la relation prédicative. Résultat : un beau parcours. Ce n'est pas un hasard si les composés en WH- et -EVER n'apparaissent jamais dans les questions, ni comme relatifs.

(9) 'which restaurant?'-'whichever you prefer'6

(10) come at 2 or 2.30 , whichever suits you best

(11) it won't matter whichever hotel we go to

On voit qu'avec WHICHEVER en (9), l'énonciateur réintroduit un ensemble de valeurs possibles, en neutralisant toute divergence qualitative de son point de vue: la seule propriété pertinente par rapport à la relation en cours est celle qui est commune, i.e. "(that) you prefer». On a le même phénomène en (10) et (11), où l'énonciateur pose qu'une valeur est à valider à partir d'un choix de valeurs pré-construites, mais que de son point de vue, elles sont équivalentes, ce qui l'empêche d'en valider une plutôt que l'autre.

\section{Savoir si un quantifieur est un opérateur de PARCOURS ou non}

Dans la littérature, le recours au parcours est particulièrement fréquent quand on traite des quantifieurs (ils opèrent sur des domaines discrétisés, et ils peuvent véhiculer de l'altérité ou de l'indéfini).

Or, tous les quantifieurs n'ont pas les mêmes spécificités distributionnelles, et j'ai voulu voir le lien entre ces comportements et l'éventuelle opération de parcours.

Je rappelle que pour moi, l'opération de parcours consiste à poser comme non-aboutie, la sélection d'une valeur à partir d'un domaine discrétisé. Cette absence de sélection découle d'un manque de particularisation qualitative des valeurs dans le cadre de la relation prédicative (donc les valeurs ne se distinguent pas assez pour permettre la validation d'occurrence).

36 Je vais donc opposer d'un côté les quantifieurs, dont le travail sur la classe aboutit à une validation d'occurrence (s) (EACH, BOTH, SOME, ALL); de l'autre les quantifieurs dont le travail sur la classe empêche la validation d'occurrence ( ANY, EITHER). Puis je me prononcerai sur EVERY.

Partons de EACH :

(12) A cartogram is a map on which each country is shown with its size proportional

to its population. (wwv.econ.ubc.ca)

En (12), le singulier nous rappelle le singulier de « A dog is a mammal », puisque dans les deux cas, l'élément exprimé par le sujet sert dans l'énoncé de représentant d'une classe. Mais tandis qu'avec A, cette valeur découle de l'association avec les déterminations du prédicat, avec $\mathrm{EACH}$, c'est le quantifieur même qui construit l'occurrence, en 12 de country, en tant que valeur spécifiée dans une classe finie (Chuquet :1997) et identifiée aux autres valeurs (cf les autres pays connus).

On retrouve le même type d'opération avec BOTH.

(13) Both my brothers are pains when it comes to me dating. ( www.mystickwick.com) 

a une fonction globalisante, qui ne permet pas de préconstruire la singularité des occurrences, comme c'est le cas avec EACH et BOTH. D'où l'absence de fléchage et les emplois génériques possibles de ALL (All power is dangerous...)

\begin{tabular}{|l|l|l|l|l|l|}
\hline & opération & $\begin{array}{l}\text { Classerde de } \\
\text { valeurs définies }\end{array}$ & $\begin{array}{l}\text { Emploi } \\
\text { générique }\end{array}$ & $\begin{array}{l}\text { Identification } \\
\text { Ensemble des valeurs } \\
\text { occurrences validées }\end{array}$ & $\begin{array}{l}\text { Quantifieur } \\
\text { flottant } \\
\text { (pas d'emploi } \\
\text { pronominal de } \\
\text { reprise) }\end{array}$ \\
\hline Each & Fléchage & Oui & Non & Oui & Oui \\
\hline Both & Fléchage & Oui & Non & Oui & Oui \\
\hline Some & Extraction & Non & Oui & Non & Oui \\
\hline All & Extraction & Non & Oui & Oui & \\
\hline
\end{tabular}

Ce tableau récapitulatif montre :

- que la possibilité d'un emploi générique est bloqué à chaque fois qu'un opérateur a comme argument une classe de valeurs définies,

- que la possibilité d'un emploi pronominal de reprise, comme dans le cas des quantifieurs flottants, dépend de la relation d'identification entre les valeurs validées et les valeurs de départ. 
Avec ANY et EITHER que l'on trouve aussi comme pronom dans I don't want any, Either will do ? il n'y a pas de restriction qualitative du domaine, mais un hiatus est construit entre les valeurs du domaine et l'extension quantitative non instanciée. C'est dans ce sens que l'on peut parler de partition avec ANY et EITHER. Ainsi, leur emploi comme pronom qualitativement «vide» diffère de l'emploi pronominal de BOTH par exemple qui n'implique pas de partition mais assure une reprise avec identification stricte. Pour qu'il y a pronom de reprise il fait avoir déjà construit une occurrence, ce qui est incompatible avec l'opération de parcours. On en arrive au tableau suivant :

\begin{tabular}{|l|l|l|l|l|l|}
\hline & Opération & $\begin{array}{l}\text { Classe } \\
\text { de } \\
\text { valeurs } \\
\text { finies }\end{array}$ & $\begin{array}{l}\text { Emploi } \\
\text { générique }\end{array}$ & $\begin{array}{l}\text { hiatus } \\
\text { entre l'ensemble des valeurs et } \\
\text { extension quantitative visée }\end{array}$ & $\begin{array}{l}\text { Quantifieurs } \\
\text { flottants }\end{array}$ \\
\hline Any & Parcours & Non & Oui & Oui & Non \\
\hline Either & parcours & Oui & non & Oui & Non \\
\hline
\end{tabular}

Et EVERY ? Difficile de se prononcer sur ce marqueur qui n'est jamais pronom de reprise (cf. EACH), ni partitif (cf. ANY). Cette observation, ajouté au fait que EVERY exige l'accord singulier du verbe, semble indiquer qu'il ne renvoie pas à un élément stabilisé, et qu'il ne construit pas d'extension qualitative (pas d'occurrence visée). A partir d'une classe, EVERY impliquerait la détermination d'un élément en tant qu'il est identifié aux autres éléments non définis de la classe. Contrairement à ce qui se passe avec $\mathrm{EACH}$, on aurait un renvoi à un domaine de valeurs non pas définies mais imaginaires (on comprend alors l'ordre de each and every country, et la comparaison each in turn / *every in turn), sans contrairement à ANY, qu'une extraction ne soit envisagée. On pourrait alors rapprocher cette opération de constitution d'une classe discrétisée de celle vu plus haut au sujet de OR.

\section{En conclusion : propriétés attendues d'un opérateur de PARCOURS}

Il y a beaucoup d'autres choses à dire sur les quantifieurs, mais le but n'est pas de les passer tous en revue ici, dans tous leurs emplois. Le but était de mettre en place une définition stable du PARCOURS tout en "parcourant" les différents modes de construction de la classe.

On a vu que certaines propriétés compatibles avec le parcours se dégagent et que l'on peut prédire qu'un marqueur sera opérateur de parcours s'il possède les propriétés nécessaires mais pas suffisantes suivantes:

- il véhicule une certaine indéfinitude (mais tous les marqueurs de l'indéfini ne sont pas des opérateurs de parcours),

- il renvoie à un domaine discrétisé (mais tous les marqueurs qui renvoient à ce type de domaine ne sont pas des opérateurs de parcours) 
- il construit une neutralisation au niveau prédicatif de l'altérité qualitative du domaine (mais tous les marqueurs de neutralisation qualitative ne sont pas des opérateurs de parcours)

- il n'est pas pronom de reprise, (autrement, ce n'est pas un opérateur de parcours)

Et puis, on pourrait ajouter, qu'en anglais si un marqueur contient EVER dans son étymologie, il y a des chances pour qu'on ait affaire à un opérateur de parcours, mais rien en fait n'est garanti, tout dépend de la place du morphème EVER dans le marqueur en question.

Enfin, on a vu qu'il y avait quatre modes de référence explicite de la classe :

- la construction d'un ensemble de valeurs discrétisées (every, or) sans qu'une validation quelconque soit envisagée

- la construction d'un ensemble de valeurs discrétisées à partir duquel on ne peut sélectionner une valeur pour l'associer à une extension quantitative (parcours : either, any, wh-ever...)

- la construction d'un ensemble de valeurs discrétisées à partir duquel on extrait une ou plusieurs occurrences (extraction : some, all...)

- le renvoi à un ensemble d'occurrences déjà définies (fléchage : each, both...).

\section{BIBLIOGRAPHIE}

Adamczewski, Henri, 1982, Grammaire linguistique de l'anglais, Paris, Armand Colin.

Bouscaren Janine, Persec Sylvie. \& Al., 1998, Analyse grammaticale dans les textes, Anglais : concours, Ophrys, Gap.

Chevillet, François, 1977, « Les relatifs indépendants en anglais contemporains », CIEREC XXIX, université de Saint-Etienne.

Chuquet, Jean, 1997, 'Each, every et la délimitation d'occurrences », in Cahiers de Recherche, t. 7, Gap, Ophrys.

Cotte, Pierre et Al. 1993, Les théories de la grammaire anglaise en France, Paris, Hachette Supérieur.

Culioli, Antoine, 1990-1999, Pour une linguistique de l'énonciation, Opérations et représentations, t. 1, t. 2, t. 3 Gap, Ophrys.

Culioli, Antoine, Notes de séminaires 1983-1984.

Danon-Boileau, Laurent, 1983, « This, That, Which, What » et la construction de références, in Méthodes en linguistique anglaise, CIEREC XXXIX, université de Saint-Etienne

Deschamps, Alain, 1997 « Faut-il rétablir le duel ? Etude énonciative de both, either, neither » in Cahiers de Recherche, t. 7, Gap, Ophrys.

Girard, Geneviève, 2001, « Propositions enchâssées en WH- : interrogatives indirectes, relatives nominales, ou troisièmes type de proposition? ", in GRAAT, p. 79-88.

Gournay, Lucie, 2003, «WHEN dans les énoncés du type : I'd just turned on the ignition when there was a big flash », in La subordination en anglais, A. Celle et S. Gresset (éds), Presses universitaires du Mirail. 
Gournay, Lucie, 2003, « Remarques sur les emplois propositionnels des marqueurs en WH- : une perspective énonciative ", communication à la journée sur WH-, Université de Rouen, disponible en ligne sur le site de l'ALAES.

Gournay, Lucie, à paraître, « (entre autres choses) Pourquoi les marqueurs en WH- ne sont finalement pas des opérateurs de parcours ", publication, suite à communication, colloque SAES 2004.

Gresset, Stéphane, 1984, "That/which marqueurs de relatives en anglais contemporain ", in Cahiers de recherche en grammaire anglaise, tome II, p. 202-272, Ophrys, Gap.

Khalifa, Jean-Charles, 1999, La Syntaxe aux concours, CAPES/Agrégation, théorie et pratique de l'énoncé complexe, Armand Colin, Paris.

Lancri, Annie, 1993a «L'ordre des quantifieurs flottants », in L'Ordre des mots, Domaine anglais. Université de Saint-Etienne, CIEREC, Travaux LXXVI, 121-140.

Lapaire, Jean-Rémi, Rotgé, Wilfrid, 1991, Linguistique et grammaire de l'anglais, Presses universitaires du Mirail, Toulouse.

Le Goffic, Pierre, 1994, «Indéfinis, interrogatifs, relatifs (termes en QU-) : parcours avec ou sans issue », Faits de Langues, ${ }^{\circ}$ 4, p. 31-40 PUF.

Mazodier, Catherine 1997, «I must have read it in some article : instabilité qualitative de some + discontinu singulier » in Cahiers de Recherche, t. 7, Gap, Ophrys.

Mauroy, Régis, 2003, «Quel Wh- dans les interrogatives, les relatives et les clivées », in La subordination en anglais, A. Celle et S. Gresset (éds), Presses universitaires du Mirail.

Mérillou, Catherine et Ranger, Graham, 2000, « Repérage, déformabilité et ajustement dans les propositions circonstancielles en when ", Les Cahiers FORELL, ${ }^{\circ}$ 14, CERLIP, Université de Poitiers.

Quirk, Randolph. et al. (1985) A Comprehensive Grammar of the English Language, London, Longman.

Wyld, Henry, 2001, Subordination et Enonciation, Cahiers de recherche, numéro spécial, Gap, Ophrys.

\section{NOTES}

1. Deux autres citations sur le parcours que je trouve opposées. Mes références sont les tomes de Pour une linguistique de l'énonciation, qui regroupent des articles écrits sur un laps de temps importants (1960-95) et les flottements repérés sont les conséquences normales d'un travail en perpétuel renouveau. Ma critique ne porte pas tant sur les travaux de Culioli que sur la reprise passive par d'autres de concepts qui sont en devenir.

Culioli $1990: 183$ : «Scanning occurs in questions, to take an obvious case : it implies that you're unable to find the right answer, as you scan (or survey) the abstract totality of possible answers" Culioli 1990 : 110 à propos de l'interrogation "l'énonciateur demande à autrui de parcourir les possibles afin de distinguer et de dire la valeur adéquate que l'énonciateur est lui incapable de distinguer. " Finalement, dans les questions le parcours est repéré par rapport à quel point de vue, celui de l'énonciateur ou celui d'autrui?

2. Quand ANY est suivi d'un discontinu pluriel, l'extension quantitative a toujours les propriétés d'unicité soulignées ici : Are there any linguists around here ? L'opération de ANY porte non pas sur la notion LINGUIST mais sur la notion de QUANTITE de LINGUISTS (ce qui est marqué par le 
pluriel). Une «quantité » est envisagée sans qu'une occurrence qualitativement distinguée vienne instanciée cette extension.

3. ?John or Mary is coming / Either John or Mary is coming

4. Le fait qu'à la question WHO is coming? on puisse répondre Peter, John, Mary etc. ne nous dit rien sur ce qui est construit par l'énonciateur. Dans Who is coming? l'énonciateur réfère à une occurrence, certes indéterminée qualitativement dans la classe des animés humains mais distinguée et validée dans la relation construite.

5. Comme l'a montré Danon-Boileau 1984 les propriétés qualitatives de l'élément en WH- sont signalées par ce marqueur comme repérées dans une autre relation prédicative. Que celle-ci soit déjà donnée (on aura tous les emplois avec «antécédents») ou qu'elle soit préconstruite par l'énonciateur (là, c'est le cas des questions)

6. Exemples tirés de mon dictionnaire électronique Hachette/Oxford.

\section{RÉSUMÉS}

Le concept de parcours est un bon exemple pour illustrer les problèmes liés à la métalangue dans la Théorie des Opérations Prédicatives et Enonciatives, mais c'est aussi un bon prétexte pour parler des façons de référer à une classe. En effet, si le parcours, en tant que concept métalinguistique, n'est pas toujours très stable, il se dégage un consensus selon lequel cette opération de détermination se retrouve dès que l'on renvoie à une classe ou ensemble de valeurs possibles, comme dans Any questions? (référence aux occurrences de la classe) ou Any question? (référence aux valeurs possibles de l'occurrence). Je vais montrer que ce consensus n'est pas satisfaisant, et que de toutes les façons que l'on dispose pour référer à une classe, l'opération de parcours, n'est qu'un outil parmi d'autres. Pour cela, je vais d'abord expliquer ce que j'entends par «parcours ». Dans cette étude, je m'intéresserai à plusieurs marqueurs appelés dans la littérature opérateurs de parcours : any, or, either, which, each, every... et je montrerai qu'à partir du moment où l'on se fonde sur une définition précise de l'opération de parcours, on arrive à expliquer certaines compatibilités et incompatibilités entre ces marqueurs dans le cadre de la référence à classe.

The concept of scanning is a good start to illustrate metalinguistic problems inside the Théorie des Opérations Prédicatives et Enonciatives. It's also a useful concept when talking about the process of class reference. In fact, if there are various definitions of scanning more or less divergent, it's usually taken for granted that this particular enunciative operation allows the construction of a class or a set of potential values. For instance, in "any questions?" the speaker refers to a class of occurrences, and "in any question?" the speaker refers to set of possible values for one occurrence. I argue against this consensual view, showing that scanning is just only one enunciative operation among others that allow class reference. Of course, my claim will require that I explain what the proper definition of scanning should be. In this study, I discuss a large sample of markers traditionally called scanning operators: any, or, either, which, each, every... in order to sort them out and explain some of their compatibilities or incompatibilities when class reference is at stake. 
INDEX

Keywords : which, or, either, quantifiers, indefiniteness, class, Culioli, paradox of scanning, extraction, pinpointing

Mots-clés : which, or, either, quantifieurs, indéfini, classe, Culioli, paradoxe du " parcours ", extraction, fléchage.

\section{AUTEUR}

LUCIE GOURNAY

UFR Charles V, Paris 7 